Comment. Math. Helv. 78 (2003) 555-583

0010-2571/03/030555-29

DOI $10.1007 / \mathrm{s} 00014-003-0763-\mathrm{z}$
(C) 2003 Birkhäuser Verlag, Basel

Commentarii Mathematici Helvetici

\title{
Hyperbolic Coxeter groups of large dimension
}

Tadeusz Januszkiewicz and Jacek Świątkowski

\begin{abstract}
We construct examples of Gromov hyperbolic Coxeter groups of arbitrarily large dimension. We also extend Vinberg's theorem to show that if a Gromov hyperbolic Coxeter group is a virtual Poincaré duality group of dimension $n$, then $n \leq 61$.

Coxeter groups acting on their associated complexes have been extremely useful source of examples and insight into nonpositively curved spaces over last several years. Negatively curved (or Gromov hyperbolic) Coxeter groups were much more elusive. In particular their existence in high dimensions was in doubt.

In 1987 Gabor Moussong [M] conjectured that there is a universal bound on the virtual cohomological dimension of any Gromov hyperbolic Coxeter group. This question was also raised by Misha Gromov [G] (who thought that perhaps any construction of high dimensional negatively curved spaces requires nontrivial number theory in the guise of arithmetic groups in an essential way), and by Mladen Bestvina [B2].

In the present paper we show that high dimensional Gromov hyperbolic Coxeter groups do exist, and we construct them by geometric or group theoretic but not arithmetic means.
\end{abstract}

Mathematics Subject Classification (2000). 20F55, 20F65, 20F67, 51F15, 57P10.

Keywords. Coxeter group, Gromov hyperbolic group, Poincaré duality, dimension.

Theorem 1. For any $n$ there exists a right angled Coxeter group $(W, S)$ of virtual cohomological dimension $\operatorname{vcd}(W)=n$ which does not contain $Z \oplus Z$ and thus is Gromov hyperbolic.

It is known by work of Mike Davis [D2] and Moussong [M] that this theorem reduces to the existence of a simplicial complex $L_{n}$ which is (i) flag, (ii) contains no empty square and (iii) satisfies certain homological condition (which is implied by say requiring that $L_{n}$ is an oriented pseudomanifold of dimension $n-1$ ). Such a complex gives rise to the Coxeter group $(W, S)$ with generators indexed by vertices of $L_{n}$ and relations $\left(x_{i} x_{j}\right)^{2}=1$ iff vertices $i, j$ are connected by an edge. Then (i) implies that the nerve of $(W, S)$ is $L_{n}$, (ii) implies hyperbolicity and (iii) implies that $\operatorname{vcd}(W)=n$. 
We construct simplicial complexes required for the proof of Theorem 1 using complexes of groups technique of $[\mathrm{BH}]$. The resulting spaces are interesting on their own right and we study them further in a forthcoming paper. Let us mention here only that the $L_{n}$ we construct turn out to be $K(\pi, 1)$ spaces.

Gromov hyperbolic Coxeter groups of large dimension are very different from fundamental groups of closed aspherical manifolds. For example we have

Theorem 2. If $(W, S)$ is a virtual Poincaré duality Coxeter group of dimension $n$ which does not contain $Z \oplus Z$ as a subgroup (equivalently, which is Gromov hyperbolic) then $n \leq 61$. Moreover, if $(W, S)$ is right angled, the bound improves to $n \leq 4$ and is sharp.

This theorem is an extension of a result of Ernest Borisovich Vinberg [V] (which motivated Moussong's question) which states that if there is a cocompact reflection group action on the Lobačevski space of dimension $n$ then $n \leq 29$. Vinberg presents also a significantly easier proof of a weaker bound $n \leq 61$, which we follow closely. It was observed already by Moussong $[\mathrm{M}]$ that Vinberg's result uses only Gromov hyperbolicity. The new ingredient we use to obtain the present form of the Theorem is a recent result of Davis on virtual Poincaré duality Coxeter groups [D1].

The right angled case of Vinberg theorem has the following corollary dealing with arbitrary cubical complexes.

Corollary. If $M$ is a manifold of dimension greater than 4, then it does not admit a negatively curved piecewise hyperbolic cubical metric.

The content of the paper is as follows.

Section 1 contains a necessary background on Coxeter groups.

Section 2 deals with the nonexistence results. We prove Theorem 2 following closely Vinberg's paper, discussing only points which are specific to our situation. We do not claim much originality here. The reader should be ready to consult [V]. We also establish a slightly stronger version of the Corollary.

In Section 3 we recall the simplex of groups construction in the form we need. We focus on the properties of (multi-) simplicial complexes arising as developments (coverings) of simplices of groups.

In Section 4 we define (and establish properties of) special complexes of groups which we call retractible. This is done by enriching the structure of a complex of groups with additional maps: retractions. We found functorial language very convenient here.

Section 5 contains the discussion of a further restriction on the complexes of groups. It is a secondary form of retractibility and we call it extra retractibility. The main result is Proposition 4.12 stating that extra retractibility implies the no-square condition on the associated complexes $L$. 
Section 6 contains a construction of extra retractible complexes of groups in arbitrary dimension, thus concluding the proof of Theorem 1.

The final three short sections contain variations and applications of the main result. In Section 7 we show how to construct many $C A T(-1)$ cubical complexes by a hyperbolization procedure which uses complexes $L_{n}$ constructed in the previous section.

Section 8 shows how to obtain non right-angled Gromov hyperbolic Coxeter groups from right-angled ones without changing dimension.

Finally, Section 9 addresses the issue of complexity. The complexes $L_{n}$ we construct are strikingly large. We establish a lower bound on the complexity of simplicial flag no-square pseudomanifold of dimension $n$ in general. While this is much smaller than the complexity of our examples, it is still fairly large.

We also want to call readers attention to [MS], where $C A T(-1)$ pseudomanifolds of large dimensions are constructed by a different (but related) method.

Acknowledgements. We thank Jan Dymara for useful discussions and the referee for careful reading of the manuscript.

\section{Background on Coxeter groups}

We present the necessary material concisely, since all of it is well documented in the literature, see for example [D2].

\subsection{Generalities}

Let $M=\left(m_{s t}\right)_{s, t \in S}$ be a symmetric matrix with diagonal entries equal 1 and off diagonal entries in $\{2,3, \ldots, \infty\}$. We associate with $M$ a Coxeter system $(W, S)$, where $W$ is a group, $S$ is the set of involutions $s^{2}=1$ generating $W$ and the nontrivial relations are $(s t)^{m_{s t}}=1$. If $m_{s t}=\infty$, there is no relation between $s$ and $t$ implied.

Let $A$ be a finite graph, that is a symmetric, anti-reflexive relation on the vertex set $S$. Define a matrix

$$
M^{A}=\left(m_{s t}^{A}\right)_{s, t \in S}= \begin{cases}1 & \text { if } s=t \\ 2 & \text { if } s, t \text { are connected by an edge in } A \\ \infty & \text { otherwise }\end{cases}
$$

The Coxeter system $\left(W^{A}, S\right)$ thus obtained is called the right angled Coxeter system associated with the graph $A$. When using the usual notation of CoxeterDynkin diagrams for Coxetex systems, one obtains $A$ as the dual to the CoxeterDynkin diagram of $M^{A}$, which has an edge marked $\infty$ for any pair of noncommuting generators. 


\subsection{The nerve and the Davis complex}

Subgroups generated by subsets of $S$ are called standard parabolic subgroups. The parabolic subgroup generated by $T \subset S$ is denoted $W_{T}$. Finite standard parabolics (excluding the trivial group) ordered by inclusion form a partially ordered set. Its geometric realization $[\mathrm{BH}, \mathrm{p} .370]$ is called the nerve and denoted $N(W, S)$. A coarser simplicial structure on the nerve will be useful: its vertices are elements of $S$ while a subset $T \subset S$ spans a simplex iff $T$ generates a finite subgroup of $W$.

For right angled systems the nerve has a simple description in terms of the graph $A$. Namely form a simplicial complex $A^{f}$ for which $A$ is the one skeleton, and whose higher dimensional simplices correspond to complete subgraphs in $A$. Thus one completes $A$ by filling in (inductively) a simplex if we see its boundary in the part of the complex already constructed. The result is the coarse simplicial structure of the nerve. The nerve $N(W, S)$ is the barycentric subdivision of $A^{f}$.

A simplicial complex $K=\left(K_{(1)}\right)^{f}$ obtained as above from its one skeleton $K_{(1)}$ is called a flag complex.

There is a related poset, consisting of left cosets $g W_{T}$ of all finite parabolics (this time including the trivial one), ordered again by inclusion. Its geometric realization is called the Davis complex of $(W, S)$ and is denoted by $|W|$. It is a truncated version of the Coxeter complex of $(W, S)$. Clearly $W$ acts on $|W|$ on the left (with a strict fundamental domain, [BH, p. 372]). The quotient of $|W|$ by $W$ is the cone over the nerve $N(W, S)$.

\subsection{Nonpositive and negative curvature}

Since the simplicial cone over the barycentric subdivision of any simplicial complex carries a natural cubical structure, the Davis complex of a Coxeter group can be considered as a cubical complex. Thus it carries a geodesic metric, obtained by declaring each $k$-cube to be isometric with $[0,1]^{k}$.

A fundamental observation, due to Gromov, is that a cubical complex is locally $C A T(0)$ (that is it is nonpositively curved in the comparison sense of Alexandrov and Toponogov) iff the link of any vertex is a flag complex. Moussong [M] amplified this observation, and showed what is the right choice of metrics on cubes yielding a $C A T(0)$ metric for an arbitrary Coxeter group. Morevover, he showed that if there is no "obvious" obstruction then this metric can be modified to a (W-invarint) $C A T(-1)$ metric on $|W|$. The obvious obstruction arises when either $(W, S)$ contains an affine Coxeter system of rank at least 2 as a standard parabolic subgroup, or it contains a standard parabolic which is a product of two infinite Coxeter systems. Thus a right angled Coxeter group acts isometrically and cocompactly on a $C A T(-1)$ space iff it does not contain a product of two infinite dihedral groups as a standard parabolic. This condition is equivalent to the following combinatorial fact: If $a, b, c, d$ are vertices of $A$ forming a square, ie. there are edges $a b, b c, c d, d a$ in $A$, then at least one of the edges $a c, b d$ is in $A$.

We say that a flag simplicial complex $A^{f}$ as above contains no empty square 
or that it is a no-square complex.

\subsection{The virtual cohomological dimension}

The virtual cohomological dimension of a Coxeter group, denoted $v c d(W)$, has been computed by Bestvina [B1], in the form of an inductive algorithm giving the answer. A clearer formulation is due to Davis [D1], who gave a formula for cohomology of $W$ with coefficients in the group ring (which gives the dimension). Namely

$$
v c d(W, S)=\max \left\{n: H^{n}\left(D, D^{T} ; Z\right) \neq 0\right\}=1+\max \left\{n: \widetilde{H}^{n}\left(D^{T} ; Z\right) \neq 0\right\}
$$

Here the maximum is taken over all $T \subset S$ such that $W_{T}$ is finite, $D$ denotes the cone over the nerve $N(W, S)$, and $D^{T}$ is the union of closed simplices of $N(W, S)$ which contain as a vertex a singleton $s \in S-T$. The maximum over the empty set is -1 .

If $T$ spans a finite parabolic subgroup of $W, D^{T}$ is homotopy equivalent to the nerve of the parabolic subgroup spanned by $S-T$. The homotopy equivalence is obtained by deformation retraction of $D^{T}$ onto the full subcomplex in the nerve $N(W, S)$ (with respect to the coarse simplicial stucture on this nerve) spanned by the subset $S-T$ of vertices.

Thus

$$
v c d(W, S)=1+\max \left\{n: \widetilde{H}^{n}\left(N\left(W_{S-T}, S-T\right) ; Z\right) \neq 0\right\} .
$$

Notice that if $(W, S)$ is a right angled Coxeter group, whose nerve is a closed orientable pseudomanifold of dimension $n$ (that is if each simplex of dimension $(n-1)$ is contained in exactly two simplices of dimension $n$, and the manifold obtained by removing simplices of codimension 2 is orientable), then $\operatorname{vcd}(W)=$ $n+1$.

The discussion above shows that to construct a hyperbolic Coxeter group of dimension $n+1$ it is sufficient to construct a simplicial flag complex with no empty squares, which is an orientable pseudomanifold of dimension $n$. This will be done in Sections 3-6.

\section{An extension of Vinberg's theorem}

\subsection{Coxeter groups and Poincaré duality}

A group $\Gamma$ is a virtual Poincaré duality group of dimension $n$, if for some finite index subgroup $\Gamma_{0}$, the cohomology of $B \Gamma_{0}$ satisfies the Poincaré duality [D1].

A simplicial complex $N$ is a generalized homology sphere (GHS) of dimension $k$ if it has the same homology as $S^{k}$ and if the link of each simplex in $N$ is a 
generalized homology sphere of appropriate dimension. In particular, each simplex is contained in a simplex of dimension $k$, and $N$ is a pseudomanifold.

Let $(W, S)$ be a Coxeter system such that $W$ is a virtual Poincaré duality group of dimension $n$. It is a theorem of M. W. Davis [D1] that $(W, S)$ decomposes into a product of Coxeter systems $(W, S)=\left(W_{1}, S_{1}\right) \times\left(W_{2}, S_{2}\right)$, so that $W_{2}$ is a finite group and the nerve of $\left(W_{1}, S_{1}\right)$ is a generalized homology sphere of dimension $n-1$.

\subsection{Combinatorics of triangulations of generalized homology spheres}

Let $N$ be a finite simplicial complex of dimension $(n-1)$ and let $f_{i}$ denote the number of $(i-1)$-dimensional simplices in $N\left(f_{0}=1\right)$. Define numbers $h_{i}$ by the polynomial equation

$$
\sum_{i=0}^{n} h_{i} t^{i}=\sum_{i=0}^{n} f_{i-1}(t-1)^{n-i} .
$$

A theorem of Richard Stanley $[\mathrm{S}]$ asserts that if $N$ is a generalized homology sphere of dimension $(n-1)$ then

$$
h_{i}=h_{n-i} \quad \text { and } \quad h_{i} \geq 0 .
$$

V. V. Nikulin (see [V, Prop. 6.1]) showed that these restrictions on $h_{i}$ imply that

$$
\left(\begin{array}{l}
n \\
2
\end{array}\right) \frac{f_{n-1}}{f_{n-3}}< \begin{cases}\frac{4(n-1)}{n-2} & \text { for } n \text { even } \\
\frac{4 n}{n-1} & \text { for } n \text { odd. }\end{cases}
$$

The quantity on the left hand side is the average number of $(n-1)$-simplices containing given $(n-3)$-simplex. If $n \geq 5$ the inequality implies that there exists an $(n-3)$-simplex in $N$ contained in three or four $(n-1)$-simplices.

Suppose $A=\sigma_{n-3}$ is contained in three $(n-1)$-simplices. Since $N$ is a pseudomanifold, any two of these three simplices intersect along an $(n-2)$-simplex (otherwise we would have more than three top dimensional simplices containing $A$ ). In other words, the simplex $A$ contained in three $(n-1)$-simplices produces a subcomplex $Y_{3}(A) \subset N$ on $(n-2)+3=n+1$ vertices. The three additional vertices are called "black" vertices.

Similarly, if $A=\sigma_{n-3}$ is contained in four $(n-1)$-simplices $\sigma^{0}, \sigma^{1}, \sigma^{2}, \sigma^{3}$, the argument above implies that (up to renumbering) simplices $\sigma^{i}, \sigma^{i+1}$ intersect along an $(n-1)$-simplex (taking indices mod 4 ). Thus we have a subcomplex $Y_{4}(A) \subset N$. Four vertices of $Y_{4}(A)$ outside $A$ are again called "black", and we see that they form a square.

Proof of the right angled case of Theorem 2. Let $(W, S)$ be a right angled Coxeter system whose nerve $N=N(W, S)$ is a generalized homology sphere of dimension $(n-1) \geq 4$. Recall that (by the construction of the nerve) $N$ is a flag complex. 
If $N$ contains $Y_{3}$, it contains the one skeleton of $n$ dimensonal simplex, hence it is not of dimension $(n-1)$.

If $N$ contains $Y_{4}$, then it contains an empty square, since any additional edge between black vertices in $Y_{4}$ would produce a $n$-simplex. Thus the group $W$ contains $Z \oplus Z$ generated by the black vertices in $Y_{4}$ and is not hyperbolic.

To see that the bound is sharp, one uses the 120 cell.

Proof of the general case of Theorem 2. Vinberg considers weights $w(A, B)$ associated to flags $f=(A \subset B)$ of a codimension 2 and top dimensional simplex in a GHS of dimension $n-1$. If the following conditions are met with some constant $c$, for all simplices $A, B$ :

$$
\begin{gathered}
w(B)=\sum_{A \mid A \subset B} w(A, B) \leq c n \\
w(A)=\sum_{B \mid B \supset A} w(A, B) \geq 5-|A|,
\end{gathered}
$$

then $n<8 c+6$ (Prop 6.2 of $[\mathrm{V}]$ ). Here $|A|$ denotes the number of top dimensional simplices containing $A$.

Choose the weights as follows: look at the two "black" (that is not contained in $A$ ) vertices of $B$ and compute their distance in the Dynkin diagram of $(W, S)$; if the distance is at most 7 , the weight is 1 , otherwise it is 0 .

In [V, Lemma 6.1] condition (1) is checked with $c=7$. It is a fact about any finite Coxeter system, thus holds in the present generality.

Condition (2) has to be checked only for $A$ such that $|A|=3,4$.

If $|A|=3$, first note that the subgroup $W_{Y}$ generated by the vertices of $Y_{3}(A)$ is infinite (otherwise $N$ would contain an $n$-simplex). Take a minimal infinite parabolic subgroup $L<W_{Y}$ containing all black vertices and denote the generating set inherited from $W_{Y}$ by $l$. Removing a black vertex from $l$ yields a finite subgroup by construction of $Y_{3}(A)$. Removing a white vertex yields a finite subgroup by minimality of $L$. The subsystem $(L, l)$ is irreducible since otherwise it would contain an infinite factor. Thus $L$ is either affine or hyperbolic since its associated quadratic form contains a positive definite subspace of codimension one. Since $L$ has at least 3 generators, it cannot be affine: an irreducible affine Coxeter group on $k+1$ generators contains a subgroup isomorphic to $Z^{k}$. Thus $L$ is one of the Lanner groups (i.e. cocompact reflection groups on a Lobačevskii space, whose fundamental domain is a simplex), and Vinberg showed that $w(A) \geq 2=5-|A|$.

If $|A|=4$, an argument similar to the one above shows that a minimal infinite parabolic subgroup of $Y_{4}(A)$ containing two opposite black vertices, and not containing the other pair of black vertices, is a Lanner group, and hence Vinberg's argument estimating the sum of weights $w(A) \geq 1=5-|A|$ goes through.

Since $c=7$, the bound $n<8 c+6=62$ follows and the proof is complete. 


\subsection{Examples and comments}

There are some issues related to Theorem 2 which still should be addressed. First, there is a question if the bound proved is sharp. Vinberg provides a better bound $n \leq 29$ for cocompact reflection groups acting on Lobačevski hyperbolic space; we did not try to adapt his argument to the present context.

It is quite conceivable that the maximum dimension of a Gromov hyperbolic Poincaré duality group is not attained on a Lobačevski hyperbolic group. Anyway there are many more Gromov hyperbolic Poincaré Duality Coxeter groups than Lobačevski hyperbolic.

Example 2.3.1. Let $W$ be a cocompact right angled Coxeter group on $H^{4}$ with fundamental domain (and the quotient) the simple polytope $P$. Thus faces of $P$ correspond to generators of $W$, and the relations are $s^{2}=1$ and $(s t)^{2}=1$ iff faces corresponding to $s, t$ intersect. Let $m_{s t}$ be the Coxeter matrix of $(W, S)$

We change the orbifold structure on $P$, by choosing a face of codimension 2 (thus two faces of codimension $1, s_{0}$ and $t_{0}$, containing it), and declaring the dihedral angle along that face to be $\frac{\pi}{n}$. In other words we take the Coxeter system $\left(W_{n}, S\right)$ which has the same system of generators as $W$, and the Coxeter matrix is

$$
n_{s, t}= \begin{cases}m_{s, t} & \text { if }(s, t) \neq\left(s_{0}, t_{0}\right), \\ n & \text { otherwise. }\end{cases}
$$

Coxeter systems $(W, S)$ and $\left(W_{n}, S\right)$ have isomorphic nerves, thus the Davis complex of $W_{n}$ is homeomorphic to $R^{4}$. The fundamental domain for $W_{n}$, denoted $P_{n}$, is combinatorially isomorphic to $P$.

Using Moussong's theorem we see that $W_{n}$ is Gromov hyperbolic (an elementary smoothing argument shows that there exists a $W_{n}$ invariant smooth Riemannian metric of negative curvature on $\left.\left|W_{n}\right|\right)$. We claim that $W_{n}$ does not act discretely cocompactly by isometries on $H^{4}$.

Suppose it does. Since the argument becomes simpler for $n=2 k$, we assume that. First, since the ideal boundaries of $\left|W_{n}\right|$ and $H^{4}$ are $W_{n}$-equivariantly homeomorphic (uniqueness of the boundary of a hyperbolic group), $W_{n}$ acts on $H^{4}$ as a reflection group and $H^{4}$ is equivariantly homeomorphic to $\left|W_{n}\right|$.

Consider a generator $s$ and the action of the centralizer of $s$ on the Fix $(s)=H^{3}$; we easily see that $Z_{W_{n}}(s)$ is isomorphic to $Z_{W}(s)$ (this is where the condition $n=2 k$ is useful), and their actions on $H^{3}$ are isometric.

Thus the polytopes $P, P_{n}$ are combinatorially equivalent, and their corresponding faces are isometric. This implies the fundamental domains, hence the Coxeter systems $(W, S)$ and $\left(W_{n}, S\right)$ are isomorphic, a contradiction.

This example essentially comes from [GT], where the smoothing to negatively curved metric is described. The change of the orbifold structure of the quotient is analogous to taking a ramified cover. The argument we used is an elementary 
substitute to Mostow rigidity. One can produce many more examples of this kind by changing dihedral angles along the set of disjoint codimension 2 faces of $P$. These variations seem to give an enormous supply of negatively curved 4 dimensional reflection groups.

Example 2.3.2. Higher dimensional examples are harder to construct. The largest known dimension of a cocompact Lobačevski hyperbolic Coxeter group is eight and it has been found in $[\mathrm{Bu}]$. On the other hand we have the following easy example of an eight dimensional Gromov hyperbolic Coxeter group.

Consider a Coxeter group $W$ with 10 generators, whose Dynkin diagram is an interval with labels $(5,3,3,3,3,3,3,3,5)$ on the edges. One readily checks with Moussong theorem that it is Gromov hyperbolic. The Davis complex is homeomorphic to $R^{8}$ (if instead of the 3 in the middle one puts 2 , ie. if one cuts the middle edge, the resulting group is the product of two 4-dimensional hyperbolic Lanner groups, and the two groups have the same poset of finite parabolic subgroups).

One checks with an argument similar to that in Example 2.3.1 that $W$ is not Lobačevski hyperbolic.

\subsection{Nonexistence of cubical $C A T(-1)$ metrics}

Proof of the Corollary. A piecewise hyperbolic metric on a cubical complex is a geodesic metric obtained by declaring each cube to be isometric to the symmetric cube in the Lobačevskii space of curvature -1, of some given edge length.

It is a theorem of Gromov, (a more general result is proved in [M]) that for a cubical complex $X$, there exists an edge length of the cube such that the cubical metric is locally $C A T(-1)$ iff the link of any vertex in the complex is a flag complex which satisfies the no-square condition. By the discussion in Section 1 existence of such a metric on a manifold of dimension $n \geq 5$ implies the existence of a $C A T(-1)$ right-angled Coxeter group, for which the Davis complex is an $n$-manifold. This contradicts Theorem 2.

Observe that we indeed proved a stronger result:

Proposition. 2.4.1. Let $X$ be a $C A T(-1)$ cubical complex and let $c$ be a cube of codimension $(k+1)$ (so that the link of $c$ has dimension $k$ ). If $k \geq 4$, then the link of $c$ is not a generalized homology sphere.

Proposition 2.4.1 shows that not every link can arise in a $C A T(-1)$ cubical complex. Simple arguments with subdivision [Dr] show there is no such obstruction for links of dimension $\leq 2$. It is unclear to us what happens for links of dimension 3. It would also be interesting to obtain further restrictions on links in higher dimensions.

Remark. One should not become too enthusiastic about the Corollary. It is very 
likely that many manifolds with locally $C A T(0)$ cubical metrics have Gromov hyperbolic fundamental groups and carry (noncubical) $C A T(-1)$ metrics. Examples of this kind are known to us in low dimensions.

\section{Simple complexes of groups}

We will construct complexes required for the proof of Theorem 1 as normal covers of certain simple complexes of groups. Such complexes are necessarily fairly large, and we view their symmetry groups as an efficient bookkeeping device. Recall first quickly the formalism of simple complexes of groups [BH, II.12].

Let $(\mathcal{Q}, \leq)$ be a partially ordered set; consider it as a small category with an object for each element in $\mathcal{Q}$ and a unique morphism $S \rightarrow T$ for any pair of elements with $S \leq T$. A simple complex of groups over $\mathcal{Q}$, denoted $G(\mathcal{Q})$, is a functor from $\mathcal{Q}$ to the category of groups and injective maps. A complex is developable if all the groups $G_{S}$ inject into the direct limit $\widehat{G(\mathcal{Q})}$.

For a developable complex together with a homomorphism $\phi: \widehat{G(\mathcal{Q})} \rightarrow G$, injective on local groups $G_{S}$, one performs The Basic Construction ([BH, II.12.18]). It gives a space $D(\mathcal{Q}, \phi)$ called the development of $G(\mathcal{Q})$ associated to $\phi$. The group $G$ acts on $D(\mathcal{Q}, \phi)$ with a strict fundamental domain $|\mathcal{Q}|$, which is isomorphic to the geometric realization of the poset $\mathcal{Q}$. The isotropy subgroups of points in the fundamental domain are the local groups $G_{S}$.

\subsection{Developable simplices of groups}

For a finite set $S$, let $\Delta_{S}$ be the simplex dual to $S$, i.e. the simplex of dimension $|S|-1$, with codimension one faces labeled by elements of $S$ and denoted $\sigma_{s}: s \in S$. For a nonempty subset $T \subset S$ we put $\sigma_{T}=\sigma_{T}^{S}:=\cap_{s \in T} \sigma_{s}$. We also use the convention that $\sigma_{\emptyset}=\sigma_{\emptyset}^{S}:=\Delta_{S}$. For vertices $\sigma_{S-s}$ we use shorter notation $v_{s}$. In this notation, the vertex $v_{s}$ is opposite to the face $\sigma_{s}$, so that $\Delta_{S}=v_{s} * \sigma_{s}$. Moreover, any face $\sigma_{T}$ of $\Delta_{S}$ is spanned by the vertices $v_{s}: s \in S-T$. For a point $p \in \Delta_{S}$, denote by $S(p)$ the (unique) subset of $S$ such that $p \in \operatorname{int} \sigma_{S(p)}$.

Let $\mathcal{Q}=P(S)$ be the poset of all subsets of $S$ and let $F$ be a simple complex of groups over $\mathcal{Q}$. In other words, to any $T \subset S$ assign a group $F_{T}$, so that if $U \subset T$, then $F_{U} \subset F_{T}$. Groups of the form $F_{\{s\}}$ will be called singleton groups.

Restricting $F$ to the subposet $\mathcal{Q} \backslash\{S\}$ we obtain a simplex of groups $F\left(\Delta_{S}\right)$ equipped with the homomorphism $\phi_{S}: \widehat{F\left(\Delta_{S}\right)} \rightarrow F_{S}$ induced by inclusions $F_{T} \rightarrow$ $F_{S}$.

Define an equivalence relation $\sim$ on $\Delta_{S} \times F_{S}$ by

$$
(p, \gamma) \sim(q, \eta) \quad \text { iff } \quad p=q \quad \text { and } \quad \gamma^{-1} \eta \in F_{S(p)}
$$

denote by $[p, \eta]$ the equivalence class of $(p, \eta)$, let

$$
L_{S}=\Delta_{S} \times F_{S} / \sim .
$$


This is the Basic Construction of [BH, II.12] applied to the pair $\left(F\left(\Delta_{S}\right), \phi_{S}\right)$, except that we insist on using a coarser simplicial structure than $[\mathrm{BH}]$ (who use the barycentric subdivisions of our simplices).

\section{Proposition 3.2.}

(1) $L_{S}$ is a pure $^{1}(|S|-1)$-dimensional multisimplicial ${ }^{2}$ complex.

(2) The group $F_{S}$ acts on $L_{S}$ by $\gamma[p, \eta]=[p, \gamma \eta]$, and the quotient map is equal to the map induced by the projection onto the first factor. The stabilizer of the point $[p, 1]$ is $F_{S(p)}$.

(3) $L_{S}$ is finite iff the index $\left[F_{S}: F_{\emptyset}\right]$ is finite. $L_{S}$ is locally finite iff all indices $\left[F_{R}: F_{\emptyset}\right]$ for proper subsets $R \subset S$ are finite.

(4) $L_{S}$ is a closed pseudomanifold iff $\left[F_{\{s\}}: F_{\emptyset}\right]=2$ for all singleton groups $F_{\{s\}}$.

(5) $L_{S}$ is gallery connected ${ }^{3}$ iff the singleton subgroups $F_{\{s\}}$ generate $F_{S}$.

(6) $L_{S}$ is an orientable pseudomanifold iff in addition to (4) there is a homomorphism $\rho: F_{S} \rightarrow Z_{2}$ whose restriction $\rho_{s}: F_{\{s\}} \rightarrow Z_{2}$ has kernel equal to $F_{\emptyset}$ for each $s \in S$.

(7) $L_{S}$ is a simplicial complex iff $F_{R} \cap F_{T}=F_{R \cap T}$ for all subsets of $S$.

Proof. Assertions (1) and (2) are obvious. The first part of (3) follows by observing that top dimensional simplices in $L_{S}$ are indexed by cosets $F_{S} / F_{\emptyset}$. Similarly, if $\sigma$ is a simplex in $L_{S}$ whose image under the quotient map $L_{S} \rightarrow \Delta_{S}$ is $\sigma_{T}$, then the set of top dimensional simplices containing $\sigma$ is indexed by the cosets $F_{T} / F_{\emptyset}$. This implies the second part of (3). The same argument applied to simplices $\sigma$ of codimension 1 proves (4).

Assertion (5) follows by observing that simplices $\left[\Delta_{S}, g_{1}\right]$ and $\left[\Delta_{S}, g_{2}\right]$ share a codimension 1 face iff $g_{2}=g_{1} g$ for some $g$ in one of the singleton groups $F_{\{s\}}$.

To prove (6), suppose that $L_{S}$ is a pseudomanifold. Pulling back a fixed orientation of $\Delta_{S}$ by the quotient map $L_{S} \rightarrow \Delta_{S}$, we equipped top dimensional simplices of $L_{S}$ with orientations which are opposite for any pair of adjacent (i.e. sharing a codimension 1 face) simplices. Homomorphism $\rho$ as in (6) induces the map from top dimensional simplices to $Z_{2}$ with a similar property: its values at any pair of adjacent simplices are distinct. Modify the induced orientations by reversing them on simplices assigned with the nonzero value in $Z_{2}$. This makes the orientations consistent and proves one of the implications in (6). The converse follows by a similar argument.

\footnotetext{
$1 X$ is pure $n$-dimensional if any $x \in X$ is in a closure of an $n$-dimensional simplex.

2 In a multisimplicial complex there may be more than one simplex with a given vertex set, but the complex is still a sum of embedded closed simplices intersecting along sums of their faces.

3 A pure complex $X$ is gallery connected if for any two top dimensional simplices $\Delta_{1}, \Delta_{2}$ there is a sequence of top dimensional simplices $\Delta_{1}=y_{1}, y_{2} \ldots y_{k}=\Delta_{2}$, so that $y_{i}$ has a common face of codimension 1 with $y_{i+1}$.
} 
To prove (7), observe that $k$-dimensional simplices in $L_{S}$ are indexed by cosets of the form $F_{S} / F_{S-K}$, where $K$ is a $k$-element subset of $S$. Assignment of the set of its vertices to a $k$-simplex $g F_{S-K}$ can be viewed as a map $F_{S} / F_{S-K} \rightarrow$ $\prod_{s \in K} F_{S} / F_{S-\{s\}}$ mapping $g F_{S-K}$ to $g F_{S-\{s\}}$. Clearly this map is equivariant with respect to the left action of $F_{S}$. A simplex is uniquely determined by the set of its vertices iff the map $F_{S} / F_{S-K} \rightarrow \prod_{s \in K} F_{S} / F_{S-\{s\}}$ is injective. This happens iff $F_{S-K}=\bigcap_{s \in K} F_{S-\{s\}}$. The last equality is satisfied for all nonempty subsets $K$ iff $F_{R} \cap F_{T}=F_{R \cap T}$ for all subsets.

\section{Retractibility}

We start by enriching the category $P(S)$ with additional morphisms. Consider an element $1 \notin S$, called a basepoint, and put $T_{*}:=T \cup\{1\}$ for each $T \in P(S)$. As the set of morphisms from $R$ to $T$ declare the set of all maps $m: R_{*} \rightarrow T_{*}$ with the property that $m(s) \in\{1, s\}$. Denote the new category by $P_{r}(S)$.

Observe that for each morphism $m: R \rightarrow T$ we have $m(1)=1$ and $m(s)=1$ for all $s \in R-T$.

For any pair $R, T$ there is a distinguished morphism $m_{R T}: R_{*} \rightarrow T_{*}$, given by

$$
m_{R T}(s)= \begin{cases}s & \text { if } s \in R \cap T \\ 1 & \text { otherwise. }\end{cases}
$$

Morphisms $m_{R T}$ with $R \subset T$ and $T \subset R$ respectively, will be called inclusions and retractions. Since for any morphism $m: R \rightarrow T$ we have $m=m_{m(R)-\{1\}, T^{\circ}}$ $m_{R, m(R)-\{1\}}$, it follows that any morphism is a composition of an inclusion and a retraction. In particular, for any $R, T$ we have $m_{R T}=m_{T \cap R, R} \circ m_{T, T \cap R}$.

Definition 4.1. A retractible complex of groups over the poset $P(S)$ is a covariant functor $F$ from the category $P_{*}(S)$ to the category of groups.

We still denote by $F_{T}$ the group assigned by a functor to a subset $T \subset S$. For any subsets $R, T \subset S$, the homomorphism induced by the morphism $m_{R T}$ is denoted by $h_{R T}: F_{R} \rightarrow F_{T}$.

\section{Lemma 4.2.}

(1) Homomorphisms induced by inclusions $R \subset T$ are injective.

(2) Homomorphisms $h_{T R}$ with $R \subset T$ (i.e. homomorphisms induced by retractions) map the subgroup $F_{R} \subset F_{T}$ identically to itself.

(3) If $R, U \subset T$, then $\left.h_{T R}\right|_{F_{U}}=h_{U \cap R, R} \circ h_{U, U \cap R}$, and hence $h_{T R}\left(F_{U}\right)=F_{U \cap R}$. In particular, $h_{T R}\left(F_{\{s\}}\right)=F_{\emptyset}$ for $s \in T-R$.

(4) If $R, T \subset S$, then $F_{R} \cap F_{T}=F_{R \cap T}$ as subgroups of $F_{S}$.

Proof. If $R \subset T$ then by functoriality we have $h_{T R} \circ h_{R T}=i d_{F_{R}}$, which proves both (1) and (2). Property (3) follows from (1) and (2) by noting that $h_{T R} \circ$ 
$h_{U T}=h_{U \cap R, R} \circ h_{U, U \cap R}$ (which follows by functoriality from a similar identity for morphisms in the category $\left.P_{r}(S)\right)$.

To see (4), note that $\left.h_{S R}\right|_{F_{R}}=i d_{F_{R}}$ and $h_{S R}\left(F_{T}\right)=F_{T \cap R}$. Thus, if $x \in$ $F_{R} \cap F_{T}$, then $x=h_{S R}(x) \in F_{T \cap R}$, and hence $F_{R} \cap F_{T} \subset F_{R \cap T}$. The opposite inclusion is obvious.

If $F$ is a retractible complex of groups on $P(S)$, the associated complex $L_{S}$ has additional properties.

Proposition 4.3. Let $F$ be a retractible complex of groups on the poset $P(S)$ and let $L_{S}$ be the associated development resulting from the basic construction in 3.1.

(1) $L_{S}$ is a simplicial complex.

(2) If $v \in L_{S}$ is a vertex that projects to $v_{s}$ under the quotient map $L_{S} \rightarrow \Delta_{S}$, then the star st $\left(v, L_{S}\right)$ of this vertex in $L_{S}$ is a full subcomplex isomorphic to the simplicial cone over the development $L_{S-\{s\}}$ (associated to the complex of groups $F$ restricted to $P(S-\{s\}))$.

(3) $L_{S}$ is flag.

(4) If for each $s \in S$ we have $\left[F_{\{s\}}: F_{\emptyset}\right]=2$ (in particular if $F_{\emptyset}=\{1\}$ and $\left.F_{\{s\}}=Z_{2}\right)$ then $L_{S}$ is an orientable pseudomanifold.

Proof. Property (1) is an immediate consequence of Proposition 3.2(7) and Lemma $4.2(4)$.

To prove (2) we use the complex $D_{s}$ introduced in Section 5, just before Lemma 5.1. Observe that the star of $v=\left[v_{s}, g\right]$ in $L_{S}$ is equal to the translate $g D_{s}$ of $D_{s}$. By Lemma 5.2, this star is then isomorphic to the simplicial cone over $L_{S-\{s\}}$. To prove that this star is full, it is sufficient to prove that $D_{s}$ is full in $L_{S}$. Let $\tau$ be the simplex of $L_{S}$ with all vertices in $D_{s}$. By Lemma 5.1, the complex $D_{s}$ is a strict fundamental domain for the action of a subgroup $H_{s}^{S} \subset F_{S}$ on $L_{S}$. Thus the image of the simplex $\tau$ under the quotient map $L_{S} \rightarrow D_{s}$ (associated to the action of $H_{s}^{S}$ ) has the same vertices as $\tau$, and hence is equal to $\tau$. It follows that $\tau$ is contained in $D_{s}$, which finishes the proof of (2).

Please note that proofs of Lemmas 5.1 and 5.2 which we used are independent of results in Section 4.

A simplicial complex $X$ with full stars of vertices is flag iff all stars of vertices in $X$ are flag. Moreover, a cone over a flag complex is again flag. Thus one can use (2) inductively to conclude $L_{S}$ is flag. To start induction we note that 0 -dimensional complexes are flag. This proves $(3)$.

The hypothesis (4) yields a homomorphism $\rho_{s}: F_{\{s\}} \rightarrow Z_{2}$ as in Proposition 3.2 (6) for each $s \in S$. A homomorphism $\rho: F_{S} \rightarrow Z_{2}$ given by the formula

$$
\rho(g):=\sum_{s \in S} \rho_{s} \circ h_{S,\{s\}}(g),
$$

is well defined and extends all homomorphisms $\rho_{s}$ for $s \in S$, which can be easily verified using Lemma 4.2. Applying Proposition 3.2(6) finishes the proof. 
Definition 4.4. A retractible complex of groups $F$ over the poset $P(S)$ is reduced if $F_{\emptyset}=\{1\}$.

Observe that to any retractible complex of groups $F$ there is associated a reduced complex $F^{0}$ over the same poset, with groups $F_{T}^{0}:=\operatorname{ker}\left(h_{T, \emptyset}: F_{T} \rightarrow F_{\emptyset}\right)$ and with the induced homomorphisms equal to the restrictions of the corresponding homomorphisms for $F$. The development $L_{S}^{0}$ associated to $F^{0}$ is isomorphic to $L_{S}$, and the isomorphism is given by $[p, g] \rightarrow\left[p, h_{S, \emptyset}(g)\right]$.

Definition 4.5. A retractible complex of groups $F$ over the poset $P(S)$ is minimal if each of the groups $F_{T}$ is generated by the union of the singleton subgroups $F_{\{s\}}: s \in T$.

To any retractible complex of groups $F$ there is associated a minimal complex $F^{\text {min }}$ over the same poset, with groups $F_{T}^{\min }:=\left\langle\cup_{s \in T} F_{\{s\}}\right\rangle$ and with the induced homomorphisms equal to the restrictions of the corresponding homomorphisms for $F$. The development $L_{S}^{\mathrm{min}}$ associated to $F^{\mathrm{min}}$ is easily seen to be the gallery connected component of the simplex $\left[\Delta_{S}, 1\right]$ in $L_{S}$. The whole $L_{S}$ is isomorphic to the disjoint union of $F_{S} / F_{S}^{\mathrm{min}}$ copies of $L_{S}^{\mathrm{min}}$, glued along some faces of codimension at least 2 .

\subsection{Examples of retractible complexes of groups}

First examples of functors on $P_{r}(S)$ are given by direct product and free product of singleton groups. More specifically assign to any $s \in S$ a group $G_{s}$; then put $F_{T}^{\text {direct }}:=\prod_{s \in T} G_{s}$ and $F_{T}^{\text {free }}:=*_{s \in T} G_{s}$.

For another example, suppose that $S$ is a vertex set of a graph $A$. With $T \subset S$ we associate the direct limit of the poset of groups $\lim \operatorname{dir} \prod_{s \in K} G_{s}$, where $K \subset T$ spans a complete subgraph of $A$. This yields the graph product functor over $P_{r}(S)$.

Finally, suppose that we are given an infinite Coxeter matrix $\left(m_{s, t}\right)_{s, t \in S}$, with even off diagonal elements ( $\infty$ is declared an even number). Then assigning to $T \subset S$ the Coxeter group with the matrix $\left(m_{s, t}\right)_{s, t \in T}$ we get a functor on $P_{r}(S)$. The restriction to even entries is necessary to have homomorphisms induced by retractions.

The examples described above are all reduced and minimal. Note that any reduced and minimal functor $F$ on $P_{r}(S)$ is sandwiched between the free and the direct product of its singleton groups. More precisely, there are natural transformations yielding surjective homomorphisms $F_{T}^{\text {free }} \rightarrow F_{T} \rightarrow F_{T}^{\text {direct }}$ for each $T \subset S$.

\subsection{Examples of developments}

We discuss developments $L_{S}$ associated to functors from 4.6.

For $F=F^{\text {direct }}$, the complex $L_{S}$ is the simplicial join $*_{s \in S} F_{\{s\}}$. In particular, 
if all the singleton groups are $Z_{2}, F_{T}^{\text {direct }}$ is $Z_{2}^{T}$ and the complex $L_{S}$ is isomorphic to the standard hyperoctahedral triangulation of the sphere $S^{|S|-1}$. In the general case, when the singleton groups are nontrivial, $L_{S}$ is a spherical Tits building with hyperoctahedral apartments.

If $F=F^{\text {free }}$ and the singleton groups are $Z_{2}$, the complex $L_{S}$ is isomorphic to a thickening of a regular $S$-valent tree with $(|S|-1)$-dimensional simplices.

More generally, if $F$ is a graph product functor based on a graph $A$ with the vertex set $S$, the space $L_{S}$ is the Tits (simplicial) building associated with the right angled Weyl group given by the matrix $M^{A}$. The thickness of the building at the face corresponding to $s$ is equal $\left|F_{\{s\}}\right|$.

Finally, if $F$ comes from an even Coxeter matrix $M, L_{S}$ is the Coxeter complex of the Coxeter system $\left(F_{S}, S\right)$ given by the matrix $M$. Here we identify the elements $s \in S$ with the generators in the singleton subgroups $F_{\{s\}}=Z_{2}$.

\section{Extra retractibility and no-square condition}

We say that a simplicial complex $X$ contains no empty square (or satisfies the no-square condition) if for any vertices $a, b, c, d$ in $X$ connected by edges $a b, b c$, $c d$ and $d a$ (thus forming a square $a b c d$ in $X$ ) at least one of the edges $a c$ or $b d$ is in $X$.

The hyperoctahedral triangulation of $S^{|S|-1}$ mentioned in 4.7 shows that the space $L_{S}$ associated to an arbitrary retractible complex of groups may contain empty squares. Thus when trying to construct no-square flag complexes, we need further restrictions on the complex of groups. To formulate them a preliminary discussion is necessary.

We will often deal with sets of form $S \backslash\{s\}$, and we use a simplified notation $S-s$ for them. We also denote by $s^{g}$ the conjugation $g s g^{-1}$, and apply the same convention $H^{g}=g \mathrm{Hg}^{-1}$ for conjugation of subgroups.

Let $F$ be a retractible complex of groups over $P(S)$. For a subset $T \subset S$ and for any $s \in T$, denote by $H_{s}^{T}$ the kernel of the homomorphism $h_{T, T-s}: F_{T} \rightarrow F_{T-s}$. We focus attention on the groups $H_{s}^{S}$ and their actions on the complex $L_{S}$. Put

$$
D_{s}=\cup_{g \in F_{S-s}}\left[\Delta_{S}, g\right] \subset L_{S} .
$$

Lemma 5.1. The subcomplex $D_{s}$ is a strict fundamental domain for the action of $H_{s}^{S}$ on $L_{S}$, i.e. the restriction of the quotient map $q_{s}: L_{S} \rightarrow H_{s}^{S} \backslash L_{S}$ to $D_{s}$ is a bijection.

Proof. The map $j_{s}: H_{s}^{S} \backslash L_{S} \rightarrow D_{s}$ defined by

$$
j_{s}\left(H_{s}^{S} \cdot[p, \gamma]\right)=\left[p, h_{S, S-s}(\gamma)\right]
$$

is the inverse of $\left.q_{s}\right|_{D_{s}}$. 
Lemma 5.2. The complex $D_{s}$ is isomorphic to the simplicial cone $C\left(L_{S-s}\right)$, with vertex $v_{s}$ as cone vertex.

Proof. Since we have $\Delta_{S}=v_{s} * \sigma_{s}$, we write

$D_{s}=\Delta_{S} \times F_{S-s} / \sim=\left(v_{s} * \sigma_{s}\right) \times F_{S-s} / \sim \cong v_{s} *\left(\Delta_{S-s} \times F_{S-s} / \sim_{s}\right)=v_{s} * L_{S-s}$,

where $\sim_{s}$ above denotes the equivalence relation used to define $L_{S-s}$. The isomorphism in the middle comes from comparing the equivalence relation $\sim$ restricted to $\sigma_{s} \times F_{S-s}$ and the equivalence relation $\sim_{s}$. We omit the straightforward details.

We will view the complex $D_{s}$ as a stratified space: its top closed stratum is $D_{s}$ and remaining closed strata are the simplices not containing the center $v_{s}$. The boundary $\partial D_{s}$ of $D_{s}$ is the union of all nonmaximal strata $\cup_{g \in F_{S-s}}\left[\sigma_{s}, g\right]$. By Lemma 5.2, the boundary is isomorphic to the complex $L_{S-s}$. For a point $p \in D_{s}$ denote by $\sigma(p)$ the unique minimal stratum (face) of $D_{s}$ with $p \in \sigma(p)$. Lemma 5.1 implies the following.

Lemma 5.3. For each face $\sigma$ of $D_{s}$ let $\Gamma_{\sigma}$ be the stabilizing subgroup of the group $H_{s}^{S}$ at the face $\sigma$. We then have

$$
L_{S}=D_{s} \times H_{s}^{S} / \sim
$$

where $(p, \gamma) \sim(q, \eta)$ iff $p=q$ and $\gamma^{-1} \eta \in \Gamma_{\sigma(p)}$.

Now we will take a closer look at the subgroups $\Gamma_{\sigma}$.

\section{Lemma 5.4.}

(1) Each face $\sigma \subset \partial D_{\text {s }}$ has a form $\sigma=\left[\sigma_{T}, g\right]$ for some subset $T \subset S$ containing $s$ and for some $g \in F_{S-s}$.

(2) If $\sigma=\left[\sigma_{T}, g\right]$ for some $s \in T \subset S$ and some $g \in F_{S-s}$, then $\Gamma_{\sigma}=\left(H_{s}^{T}\right)^{g} \subset$ $H_{s}^{S}$.

Proof. Only the second assertion requires a proof. Observe that $\operatorname{Stab}\left(F_{S},\left[\sigma_{T}, 1\right]\right)=$ $F_{T}$, and hence $\operatorname{Stab}\left(F_{S},\left[\sigma_{T}, g\right]\right)=\left(F_{T}\right)^{g}$. Using the fact that the subgroup $H_{s}^{S}$ is normal in $F_{S}$, we have $\Gamma_{\sigma}=\operatorname{Stab}\left(H_{s}^{S},\left[\sigma_{T}, g\right]\right)=\left(F_{T}\right)^{g} \cap H_{s}^{S}=\left(F_{T} \cap H_{s}^{S}\right)^{g}=$ $\left(F_{T} \cap \operatorname{ker} h_{S, S-s}\right)^{g}=\left(\operatorname{ker} h_{T, T-s}\right)^{g}=\left(H_{s}^{T}\right)^{g}$.

From now on all retractible complexes of groups will be reduced and minimal. Under this restriction we obtain nice descriptions of various groups in terms of generators.

Lemma 5.5. If $F$ is reduced and minimal then the subgroup $H_{s}^{T}$ is generated by the union of the conjugates $\left(F_{\{s\}}\right)^{g}: g \in F_{T-s}$. 
Proof. By minimality the subgroups $F_{T-s}$ and $F_{\{s\}}$ generate $F_{T}$. Since $F$ is reduced, we have $F_{\{s\}} \subset H_{s}^{S}$. The lemma follows by combining these two facts.

Next lemma describes the subgroups $\Gamma_{\sigma}$ in terms of generators.

Lemma 5.6. Let $\sigma=\left[\sigma_{T}, \gamma\right] \subset \partial D_{s}$. Then the subgroup $\Gamma_{\sigma} \subset H_{s}^{S}$ is generated by the union of the conjugates $\left(F_{\{s\}}\right)^{g}: g \in \gamma \cdot F_{T-s}$.

Proof. Applying Lemmas 5.4 and 5.5 we have

$$
\begin{aligned}
\Gamma_{\sigma} & =\left(H_{s}^{T}\right)^{\gamma}=\left\langle h^{\gamma}: h \in \cup_{g \in F_{T-s}}\left(F_{\{s\}}\right)^{g}\right\rangle=\left\langle\cup_{g \in F_{T-s}}\left(F_{\{s\}}\right)^{\gamma g}\right\rangle \\
& =\left\langle\cup_{g \in \gamma \cdot F_{T-s}}\left(F_{\{s\}}\right)^{g}\right\rangle .
\end{aligned}
$$

Observe that the top dimensional simplices of $\partial D_{s}$ have the form $\left[\sigma_{s}, g\right]: g \in$ $F_{S-s}$ and that they correspond bijectively to the elements of $F_{S-s}$. Put $\sigma_{g}:=$ $\left[\sigma_{s}, g\right]$. The following reformulation of Lemma 5.6 will be useful later in this section.

Lemma 5.7. Let $\sigma$ be a simplex of $\partial D_{s}$. Then the group $\Gamma_{\sigma}$ is generated by the union of the conjugates $\left(F_{\{s\}}\right)^{g}: \sigma \subset \sigma_{g}$.

Proof. The inclusion $\sigma=\left[\sigma_{T}, \gamma\right] \subset\left[\sigma_{s}, g\right]=\sigma_{g}$ holds iff $\gamma^{-1} g \in F_{T-s}$ iff $g \in$ $\gamma \cdot F_{T-s}$.

Definition 5.8. A reduced, minimal, retractible complex of groups $F$ over the poset $P(S)$ is extra retractible if for each $s \in S$ and for any subset $T \subset S$ containing $s$, there is a (uniquely determined) homomorphism $r_{s, T}^{S}: H_{s}^{S} \rightarrow H_{s}^{T}$ such that:

(1) $r_{s, T}^{S}(h)=h$ if $h \in\left(F_{\{s\}}\right)^{g}$ and $g \in F_{T-s}$;

(2) $r_{s, T}^{S}(h)=1$ if $h \in\left(F_{\{s\}}\right)^{g}$ and $g \in F_{S-s}-F_{T-s}$.

Note that condition (1) above implies that $r_{s, T}^{S}(h)=h$ for each $h \in H_{s}^{T}$ and thus the homomorphism $r_{s, T}^{S}$ is a retraction. This explains why we call the property from Definition 5.8 extra retractibility.

Lemma 5.9. Let $F$ be an extra retractible complex of groups over the poset $P(S)$. Then for each simplex $\sigma \in \partial D_{s}$ there is a (uniquely determined) homomorphism $r_{\sigma}: H_{s}^{S} \rightarrow \Gamma_{\sigma}$ such that

(1) $r_{\sigma}(h)=h$ if $h \in\left(F_{\{s\}}\right)^{g}$ and $\sigma \subset \sigma_{g}$;

(2) $r_{\sigma}(h)=1$ if $h \in\left(F_{\{s\}}\right)^{g}$ and $\sigma$ is not contained in $\sigma_{\gamma}$.

Proof. Let $\sigma=\left[\sigma_{T}, \gamma\right]$. In view of Lemmas 5.6 and 5.7, the homomorphism defined by $r_{\sigma}(h):=\left[r_{s, T}^{S}\left(h^{\gamma^{-1}}\right)\right]^{\gamma}$ is as required. To see this, observe that if $h$ is as in (1) 
then $h=k^{\gamma g}$ for some $k \in F_{\{s\}}$ and for some $g \in F_{T-s}$. Thus

$$
r_{\sigma}(h)=r_{\sigma}\left(k^{\gamma g}\right)=\left[r_{s, T}^{S}\left(\left(k^{\gamma g}\right)^{\gamma^{-1}}\right)\right]^{\gamma}=\left[r_{s, T}^{S}\left(k^{g}\right)\right]^{\gamma}=\left(k^{g}\right)^{\gamma}=k^{\gamma g}=h .
$$

Similarly, one shows that $r_{\sigma}(h)=1$ if $h$ is as in (2).

\section{Remark.}

(1) Existence of homomorphisms $r_{\sigma_{g}}$ as in Lemma 5.9 implies that for distinct $g_{1}, g_{2} \in F_{S-s}$ we have $\left(F_{\{s\}}\right)^{g_{1}} \cap\left(F_{\{s\}}\right)^{g_{2}}=\{1\}$.

(2) Remark (1) above implies that the complex of groups $F^{\text {direct }}$ (for which $\left.F_{T}^{\text {direct }}=\left(Z_{2}\right)^{T}\right)$ is not extra retractible, since the groups in this complex are abelian.

(3) Slightly less clear, but still true, is the fact that the complex of groups $F^{\text {free }}$ is extra retractible. However, the groups $F_{T}$ in this complex are in general infinite and hence useless for our purpose. In Section 6 we construct a functor $F$ which is extra retractible and for which all the groups $F_{T}$ are finite.

Remark (1) above can be generalized as follows.

Lemma 5.10. Let $\sigma$ and $\tau$ be two simplices of $\partial D_{s}$ that are not contained in any common simplex of $\partial D_{s}$. Then $\Gamma_{\sigma} \cap \Gamma_{\tau}=\{1\}$.

Proof. The groups $\Gamma_{\sigma}$ and $\Gamma_{\tau}$ are generated by the unions of the families of conjugates $\left(F_{\{s\}}\right)^{g}: \sigma \subset \sigma_{g}$ and $\left(F_{\{s\}}\right)^{g}: \tau \subset \sigma_{g}$ respectively. Due to our assumption on $\sigma$ and $\tau$, these two families are disjoint. It follows that the homomorphism $r_{\sigma}$ as in Lemma 5.9 maps $\Gamma_{\sigma}$ identically to itself, and it maps all of $\Gamma_{\tau}$ to 1 . This implies that $\Gamma_{\sigma} \cap \Gamma_{\tau}=\{1\}$.

We now turn to describing the complex $L_{S}$ as tessellated by the isomorphic copies of $D_{s}$. We take as tiles the translates $h \cdot D_{s}: h \in H_{s}^{S}$ and denote the whole tessellation by $H_{s}^{S} \cdot D_{s}$. Using the description $L_{S}=D_{s} \times H_{s}^{S} / \sim$ from Lemma 5.3 , we can identify each tile $h \cdot D_{s}$ with the set $\left[D_{s}, h\right]$. It is then clear that the tiles cover all of $L_{S}$ and that they do not overlap, i.e. the intersection of any two tiles is contained in the boundary of each of them. Assuming extra retractibility, we have the following stronger

Lemma 5.11. Suppose that $F$ is an extra retractible complex of groups over the poset $P(S)$. Then the intersection of any two distinct tiles in the tessellation $H_{s}^{S} \cdot D_{s}$ is either empty or it is a single simplex contained in the boundary of each of the tiles.

Proof. Let $h_{1}, h_{2}$ be two distinct elements of the group $H_{s}^{S}$ and $\left[D_{s}, h_{1}\right],\left[D_{s}, h_{2}\right]$ the corresponding two distinct tiles. If $x \in\left[D_{s}, h_{1}\right] \cap\left[D_{s}, h_{2}\right]$ then $x=\left[p, h_{1}\right]=\left[q, h_{2}\right]$ 
and $\left(p, h_{1}\right) \sim\left(q, h_{2}\right)$. It follows that $p=q$ and $h_{1}^{-1} h_{2} \in \Gamma_{\sigma(p)}$. Thus, identifying the intersection of the tiles with a subset in $D_{s}$, we have

$$
\left[D_{s}, h_{1}\right] \cap\left[D_{s}, h_{2}\right]=\cup\left\{\sigma \in \partial D_{s}: h_{1}^{-1} h_{2} \in \Gamma_{\sigma}\right\} .
$$

Now, using homomorphisms $r_{\sigma}$ and the argument as in the proof of Proposition 4.2. (3), we see that $\Gamma_{\sigma}=\cap\left\{\Gamma_{v}: v\right.$ is a vertex of $\left.\sigma\right\}$. This implies that if all vertices of a simplex $\sigma \in \partial D_{s}$ are in the intersection of the two tiles, so is the whole $\sigma$, and thus the intersection is a full subcomplex in $\partial D_{s}$. On the other hand, all the vertices contained in the intersection have to belong to a single simplex of $\partial D_{s}$, since otherwise they span a full subcomplex in $\partial D_{s}$ containing some two simplices $\sigma$ and $\tau$ not contained in a single simplex of $\partial D_{s}$. Applying Lemma 5.10, we then get $h_{1}^{-1} h_{2} \in \Gamma_{\sigma} \cap \Gamma_{\tau}=\{1\}$, and hence $h_{1}=h_{2}$. This contradicts our initial assumption proving that the intersection is either empty or a single simplex of $\partial D_{s}$.

Proposition 5.12. Suppose that $F$ is an extra retractible complex of groups on the poset $P(S)$. Then the complex $L_{S}$ satisfies no-square condition.

Proof. Suppose there is a square in $L_{S}$ with vertices $a, b, c, d$ and with edges $a b, b c, c d$ and $d a$. By translating with an element of $F_{S}$ we may (and will) assume that $a b$ is an edge of the simplex $\left[\Delta_{S}, 1\right]$ in $L_{S}$. Let $s, t$ be the elements of $S$ such that $\left[v_{s}, 1\right]=a$ and $\left[v_{t}, 1\right]=b$. Consider the subgroup $H_{s}^{S} \subset F_{S}$ and the tessellation $H_{s}^{S} \cdot D_{s}$ of $L_{S}$. The vertex $a$ is then the unique interior vertex of the tile $D_{s}$, and we will refer to it as centre of $D_{s}$. Consider next the subgroup $H_{s}^{S-t} \subset H_{s}^{S}$ and the homomorphism $r_{s, S-t}^{S}: H_{s}^{S} \rightarrow H_{s}^{S-t}$. Define the subgroup $H_{s, t}^{S}:=\operatorname{ker} r_{s, S-t}^{S} \subset H_{s}^{S}$ and note that its restricted action on $L_{S}$ clearly preserves the tessellation $H_{s}^{S} \cdot D_{s}$. Put $D_{s, t}=\cup\left\{h \cdot D_{s}: h \in H_{s}^{S-t}\right\}$.

Claim 1. $D_{s, t}$ is a strict fundamental domain for the action of $H_{s, t}^{S}$ on $L_{S}$.

Proof of Claim 1. Viewing $L_{S}$ as $H_{s}^{S} \times D_{s} / \sim$ (see Lemma 5.3), define a map $j_{s, t}: H_{s, t}^{S} \backslash L_{S} \rightarrow D_{s, t}$ by the formula

$$
j_{s, t}\left(H_{s, t}^{S} \cdot[p, \gamma]\right)=\left[p, r_{s, S-t}^{S}(\gamma)\right] .
$$

This map is easily checked to be an inverse of the quotient map $L_{S} \rightarrow H_{s, t}^{S} \backslash L_{S}$ restricted to $D_{s, t}$, hence the claim.

Claim 2. $D_{s, t}$ is the union of all tiles of the tessellation $H_{s}^{S} \cdot D_{s}$ that contain the vertex $b=\left[v_{t}, 1\right]$.

Proof of Claim 2. Using again the description $L_{S}=H_{s}^{S} \times D_{s} / \sim$, observe that a tile $h \cdot D_{s}=\left[D_{s}, h\right]$ contains the vertex $\left[v_{t}, 1\right]$ iff $h \in \Gamma_{v_{t}}=\Gamma_{\sigma_{S-t}}=H_{s}^{S-t}$ (see Lemma 5.4). This implies the claim. 
By Claim 2 and the fact that $a$ is the centre of the tile $D_{s} \subset D_{s, t}$, the edges $a b, b c$ and $d a$ of the square $a b c d$ are contained in $D_{s, t}$. By Claim 1 , the vertices $c$ and $d$ are connected by an edge in $D_{s, t}$, namely by the image of the edge $c d$ under the quotient map $L_{S} \rightarrow H_{s, t}^{S} \backslash L_{S}=D_{s, t}$. This edge is in fact equal to $c d$, since two vertices span at most one edge in $L_{S}$.

Consider now the following two cases.

Case 1: $c \in D_{s}$. Since $D_{s}$ is a simplicial cone centered at a (see Lemma 4.2), there is an edge $a c$ in $D_{s} \subset L_{S}$. Thus the square abcd is not empty in this case.

Case 2: $c \notin D_{s}$. Let $g \cdot D_{s} \subset D_{s, t}$ be a tile containing the edge $c d$. By our assumption on $c$ we have $g \cdot D_{s} \neq D_{s}$. Next, since $a$ is the centre of $D_{s}$, we have $d \in D_{s}$ and consequently $d \in D_{s} \cap g \cdot D_{s}$. By Lemma 5.11, the intersection $D_{s} \cap g \cdot D_{s}$ is a simplex, and by Claim 2 this simplex contains the vertex $b$. It follows that there is an edge $b d$ in $L_{S}$, and again the square abcd in not empty.

\section{Construction and the proof of Theorem 1}

To prove Theorem 1 it is sufficient to construct for any natural number $n$ a flag simplicial complex $L_{n}$ of dimension $n$ which is a closed orientable pseudomanifold and contains no empty square. According to previous sections, such $L_{n}$ comes as the development $L_{S}$ of an extra retractible complex of groups $F$ on the poset $P(S)$, if $|S|=n+1$, all singleton groups are equal to $Z_{2}$ and the group $F_{S}$ is finite (Propositions 3.2, 4.3 and 5.12). We will construct such complexes of groups for any $n$. This will be done inductively, by extending the complexes of groups constructed in earlier steps to bigger posets.

Let $\left(P_{f}(A), \leq\right)$ be the poset of all finite subsets of the fixed countably infinite set $A$. View $P_{f}(A)$ as the category with inclusions and retractions in the obvious analogy with the category $P_{r}(S)$ defined at the beginning of Section 4 . As before, the induced groups are denoted by $F_{S}$, the groups $F_{\{s\}}$ are called singleton groups, and the homomorphisms induced by morphisms $m_{R T}$ are denoted $h_{R T}$.

For any morphism $F$ from the category $P_{f}(A)$ to the category of groups, and for any finite subset $S \subset A$, the restriction of $F$ to $P_{r}(S)$, denoted $F(S)$, is a retractible complex of groups. Thus we will call $F$ a retractible complex of groups on $P_{f}(A)$. We say that $F$ is reduced (minimal, extra retractible), if for any $S \in P_{f}(A)$ the restriction $F(S)$ is reduced (minimal, extra retractible, respectively). To prove Theorem 1, it is sufficient to construct an extra retractible complex of groups $F$ on $P_{f}(A)$, with all singleton groups equal to $Z_{2}$ and all groups $F_{S}$ finite. We will call such a complex of groups (or functor) wonderful.

For a natural number $n$, denote by $P_{n}(A)$ the full subcategory of $P_{f}(A)$ whose objects are all sets $S \subset A$ with cardinality $|S| \leq n$. It means that $P_{n}(A)$ contains all morphisms from the category $P_{f}(A)$ between the relevant objects. We adapt the notion of a wonderful complex of groups to functors on the categories $P_{n}(A)$ in the obvious way. A wonderful functor $F$ on $P_{f}(A)$ will be constructed by a 
sequence of extensions from a wonderful functor on $P_{n}(A)$ to a wonderful functor on $P_{n+1}(A)$.

The construction. For the first step of the induction, define $F$ on $P_{1}(A)$ in the only possible way, putting $F_{\{s\}}=Z_{2}$ for each $s \in A$. Observe that this functor is clearly wonderful. Next, assume that $F$ defined on $P_{n}(A)$ is wonderful. For each $S \subset A$ with $|S|=n+1$, denote by $F\left(\Delta_{S}\right)$ the simplex of groups (over the simplex $\Delta_{S}$ ) with local groups $F_{T}$ at faces $\sigma_{T}$ of $\Delta_{S}$. Declare $\mathcal{F}_{S}$ to be the direct limit of this simplex of groups.

Lemma 6.1. Local groups $F_{T}$ in the simplex of groups $F\left(\Delta_{S}\right)$ inject into the direct limit $\mathcal{F}_{S}$. In other words, simplex of groups $F\left(\Delta_{S}\right)$ is developable.

Proof. For a fixed local group $F_{T}$ consider the morphism from $F\left(\Delta_{S}\right)$ to $F_{T}$ given by the family of homomorphisms $h_{R T}: F_{R} \rightarrow F_{T}$ (indexed by proper subsets $R \subset S)$. This simple morphism induces a homomorphism $\mu_{S, T}: \mathcal{F}_{S} \rightarrow F_{T}$ whose composition $F_{T} \rightarrow \mathcal{F}_{S} \rightarrow F_{T}$ with the map $F_{T} \rightarrow \mathcal{F}_{S}$ (associated to the direct limit) is clearly the identity. Thus the map $F_{T} \rightarrow \mathcal{F}_{S}$ is injective.

Observe that any subset $S \subset A$ with $|S|=n+1$ identifies with the generating set for the group $\mathcal{F}_{S}$, where an element $s \in S$ represents the generator in the singleton subgroup $F_{\{s\}} \subset \mathcal{F}_{S}$ (isomorphic to $Z_{2}$ ). The fact that these generators are all distinct follows from the existence of retractions $\mu_{S,\{s\}}: \mathcal{F}_{S} \rightarrow F_{\{s\}}$ (described in the proof of Lemma 6.1). This observation is extended by the following

Proposition 6.2. The functor on the category $P_{n+1}(A)$ with groups $F_{T}$ for $|T| \leq$ $n$, and with groups $\mathcal{F}_{S}$ for $|S|=n+1$, is extra retractible.

Remark. The functor as in Proposition 6.2 satisfies all properties of a wonderful functor except that the groups $\mathcal{F}_{S}$ are in general not finite. Finite groups $F_{S}$ giving a wonderful functor on $P_{n+1}(A)$ will be defined later as certain quotients of the groups $\mathcal{F}_{S}$.

Proof of Proposition 6.2. We have first to check the existence of all homomorphisms prescribed by functoriality. Those ones which are induced by morphisms from the subcategory $P_{n}(A)$ are simply the homomorphisms given by the functor $F$. The remaining homomorphisms are obtained by composing the inclusions $F_{T} \rightarrow \mathcal{F}_{S}$ and the retractions $\mu_{S, T}: \mathcal{F}_{S} \rightarrow F_{T}$ defined in the proof of Lemma 6.1. This is so, because any morphism $m: S \rightarrow T$ in $P_{n+1}(A)$ is of the form $m=m_{m(S)-\{1\}, T} \circ$ $m_{S, m(S)-\{1\}} \cdot$ 
Since minimality and reducedness of the considered functor are obvious, we turn to proving extra retractibility. Let $S \subset A$ be a set with $|S|=n+1$. Denote by $\mathcal{H}_{s}^{S}$ the kernel of the homomorphism $\mu_{S-s}^{S}: \mathcal{F}_{S} \rightarrow F_{S-s}$. According to Lemma $5.5, \mathcal{H}_{s}^{S}$ is generated by the set $\left\{s^{g}: g \in F_{S-s}\right\}$, and the generating sets for groups $H_{s}^{T}$ with $T \subset S$ have similar form. It is sufficient to prove that for any $T \subset S$ containing $s$ there is a homomorphism $r_{s, T}^{S}: \mathcal{H}_{s}^{S} \rightarrow H_{s}^{T}$ such that

(1) $r_{s, T}^{S}(h)=h$ for each $h \in\left\{s^{g}: g \in F_{T-s}\right\}$;

(2) $r_{s, T}^{S}(h)=1$ for each $h \in\left\{s^{g}: g \in F_{S-s}-F_{T-s}\right\}$.

This requires some preparatory discussion.

Let $\mathcal{L}_{S}=\mathcal{F}_{S} \times \Delta_{S} / \sim$ be the development of the complex of groups $F\left(\Delta_{S}\right)$ associated to the direct limit $\mathcal{F}_{S}$. It follows from general theory [BH II.12] that $\mathcal{L}_{S}$ is connected and simply connected. Consider the complex of groups $\mathcal{H}_{s}^{S} \backslash \mathcal{L}_{S}$ associated to the action of $\mathcal{H}_{s}^{S}$ on $\mathcal{L}_{S}$. By lemma $5.1, \mathcal{H}_{s}^{S} \backslash \mathcal{L}_{S}$ is a simple complex of groups, and since $\mathcal{L}_{S}$ is connected and simply connected, the group $\mathcal{H}_{s}^{S}$ is the direct limit of this complex. To prove the existence of the homomorphism $\mathcal{H}_{s}^{S} \rightarrow H_{s}^{T}$ as required, it is sufficient to construct the appropriate simple morphism $\mathcal{H}_{s}^{S} \backslash \mathcal{L}_{S} \rightarrow H_{s}^{T}$, i.e. the consistent family of homomorphisms from the local groups of $\mathcal{H}_{s}^{S} \backslash \mathcal{L}_{S}$ to $H_{s}^{T}$.

According to Lemma 5.4, local groups of $\mathcal{H}_{s}^{S} \backslash \mathcal{L}_{S}$ have the form $\left(H_{s}^{R}\right)^{g}$ for the appropriate $R$ and $g$. The required homomorphisms to $H_{s}^{T}$ are then the compositions

$$
\left(H_{s}^{R}\right)^{g} \rightarrow\left(H_{s}^{R \cap T}\right)^{g} \rightarrow\left(H_{s}^{T}\right)^{g} \rightarrow H_{s}^{T}
$$

where the first map is the conjugated retraction $r_{s, R \cap T}^{R}$ (provided by extra retractibility in the inductive hypothesis), the second map is the conjugated inclusion, and the third map is the identity if $g \in F_{T-s}$ while it is the trivial map to $1 \in H_{s}^{T}$ otherwise. Conditions (1) and (2) for the induced homomorphism can be checked directly, by tracing the images of the generators under all involved maps. We omit the details.

We will now improve the functor described in Proposition 6.2 by replacing groups $\mathcal{F}_{S}$ with their appropriate finite quotients. To do this, we need certain normal subgroups of finite index in those groups. For any distinct elements $s, t \in$ $S$, denote by $\mathcal{H}_{s, t}^{S}$ the kernel of the retraction $r_{s, S-t}^{S}: \mathcal{H}_{s}^{S} \rightarrow H_{s}^{S-t}$. Observe that, since the group $H_{s}^{S-t}$ is finite, the group $\mathcal{H}_{s, t}^{S}$ has finite index in $\mathcal{H}_{s}^{S}$, and hence also in $\mathcal{F}_{S}$. Put $K_{S}:=\bigcap_{s \neq t} \mathcal{H}_{s, t}^{S}$ and normalize it to the normal subgroup $N_{S}:=\bigcap_{g \in F_{S}}\left(K_{S}\right)^{g}$ which still has finite index in $\mathcal{F}_{S}$.

Lemma 6.3. The normal subgroup $N_{S}$ is contained in the kernel of any retraction $\mathcal{H}_{s}^{S} \rightarrow H_{s}^{T}$ and any homomorphism $\mathcal{F}_{S} \rightarrow F_{R}$ induced by the functor from Proposition 6.2. 
Proof. By definition, $N_{S}$ is contained in the kernel $\mathcal{H}_{s, t}^{S}$ of any map $\mathcal{H}_{s}^{S} \rightarrow H_{s}^{S-t}$. Now, since $T \subset S-t$ for some $t$, and since the map $\mathcal{H}_{s}^{S} \rightarrow H_{s}^{T}$ is equal to the composition $\mathcal{H}_{s}^{S} \rightarrow H_{s}^{S-t} \rightarrow H_{s}^{T}$ of the retractions, the kernel of $\mathcal{H}_{s}^{S} \rightarrow H_{s}^{T}$ contains the kernel of $\mathcal{H}_{s}^{S} \rightarrow H_{s}^{S-t}$, and hence contains $N_{S}$.

The part of the lemma concerning morphisms $\mathcal{F}_{S} \rightarrow F_{R}$ has very similar proof which we omit.

The next proposition completes the inductive construction of a wonderful functor $F$ on $P_{f}(A)$, thus proving Theorem 2 .

Proposition 6.4. The functor on $P_{n+1}(A)$ obtained from a wonderful functor $F$ on $P_{n}(A)$ by putting $F_{S}:=\mathcal{F}_{S} / N_{S}$ for any $S \subset A$ with $|S|=n+1$ is wonderful.

Proof. Since by Lemma 6.3, the kernels of all homomorphisms from $\mathcal{F}_{S}$ to $F_{R}$ (induced by the functor as in Proposition 6.2) contain $N_{S}$, they induce homomorphisms from $F_{S}$ to $F_{R}$ which turn the improved functor into a reduced and minimal retractible complex of groups on $P_{n+1}(A)$. Similarly, the kernels of retractions $\mathcal{H}_{s}^{S} \rightarrow H_{s}^{T}$ all contain $N_{S}$, thus inducing homomorphisms $\mathcal{H}_{s}^{S} / N_{S} \rightarrow H_{s}^{T}$. By observing that these homomorphisms are the desired retractions $H_{s}^{S} \rightarrow H_{s}^{T}$ we get extra retractibility. Since the rest of the desired properties are obvious, the proposition follows.

\section{Hyperbolization}

Thus far we produced essentially a single $C A T(-1)$ pseudomanifold, and the reader may think that such spaces are isolated/exotic. We now show how to produce many $C A T(-1)$ cubical complexes, using hyperbolization procedure.

We start with cubical complexes which are locally $C A T(0)$, and convert them to locally $C A T(-1)$ complexes. There are several hyperbolization procedures described in [DJ1], which, starting with any cubical complex, produce a locally $C A T(0)$ one. Putting these together we end up with a hyperbolization procedure which converts any cubical complex to a locally $C A T(-1)$ one.

Let us recall [BH, II.5] that a piecewise constant curvature $\kappa$ space has curvature bounded above by $\kappa$, or satisfies the $C A T(\kappa)$ inequality locally if and only if all its links, considered as piecewise constant curvature 1 metric spaces, are large: the length of the shortest geodesic loop in any link is $\geq 2 \pi$. In particular a piecewise flat metric on a cubical complex is $C A T(0)$ iff the links are flag.

For any minimal, reduced, retractible complex of groups over $P(S)$ we have, as discussed in 4.6, a surjective map $A_{S}: F_{S} \rightarrow F_{S}^{\text {direct }}$. Assume that the singleton groups are all isomorphic to $Z_{2}$. Then this last group is isomorphic with $Z_{2}^{|S|}$, and $A_{S}$ coincides with the abelianization map. Since the complex $L_{S}^{\text {direct }}$ associated with $Z_{2}^{|S|}$ is the hyperoctahedral triangulation of the sphere, we have the associated 
map $a_{S}: L_{S} \rightarrow S^{|S|-1}$ of complexes. One can interpret this map as taking the quotient of $L_{S}$ by the kernel of $A_{S}$.

Recall that the cone over the barycentric subdivision of any simplicial complex $X$ has a natural structure of a cubical complex. We will call it the cubical cone over $X$ and denote it by $c c X$. An alternative description is as follows. For any simplex $\sigma \subset X$ we take a cube $c_{\sigma}$ of dimension $\operatorname{dim} \sigma+1$ with a distinguished vertex 0 , identify the link of 0 in $c_{\sigma}$ with $\sigma$. Now identify cubical faces containing 0 following the identifications on $X$. The cubes of this cubical structure are called small cubes in $c c X$.

Since the coning is obviously functorial, we form the cubical cone over the map $a_{S}$, to obtain a map of cubical complexes

$$
c c\left(a_{S}\right): c c L_{S} \rightarrow c c S^{|S|-1}=C^{S},
$$

with $C^{S}$, the cube $[-1,1]^{S}$, decomposed into $2^{|S|}$ images of the small cube $[0,1]^{S}$ under sign changing symmetries of the large cube.

One can view $c c L_{S}$ as a ramified covering of $C^{S}$.

We use the map $c c\left(a_{S}\right)$ as an input in a fiber product hyperbolization for cubical complexes as in [DJ1], and accordingly change the notation slightly. Now $C_{h}^{S}=c c L_{S}$ denotes the hyperbolized cube. For $C^{T}, T \subset S$, a face of dimension $|T|$ in $C^{S}$ is obtained by fixing coordinates not in $T$ to be +1 or -1 . We denote by $C_{h}^{T}$ a connected component of the preimage of $C^{T}$ by $\operatorname{cc}\left(a_{S}\right)$. It is, in fact, a cubical cone $c c L_{T}$.

Let $X$ be a locally $C A T(0)$ cubical complex.

Suppose there exists a folding map $f_{X}: X \rightarrow C^{S}$ to the $|S|$-cube $C^{S}$; the $C^{S}$ is considered here as a single cube (i.e. it is not subdivided). This means that there is a map of cubical complexes which is injective on each cube in $X$ to the $|S|$-cube (thus the complex is of dimension at most $|S|$ ).

Let $X_{h}$ be the fiber product of $f_{X}$ and $c c\left(a_{S}\right)$, that is $X \times C_{h}^{S} \supset X_{h}=$ $\left\{(x, h) \mid f_{X}(x)=c c\left(a_{S}\right)(h)\right\}$.

The intuitive content of this construction is that we replace a cube in $X$ with a copy of $C_{h}$ preserving the gluing pattern of $X$. The fiber product construction allows us to say it concisely.

The projection $X_{h} \rightarrow C_{h}$ restricted to the preimage of a top dimensional cube in $X$ under the projection $X_{h} \rightarrow X$ is an isomorphism, thus it endows $X_{h}$ with the geodesic metric, which on each $C_{h}$ is the Moussong metric.

For the reader's convenience we recall briefly Moussong's construction. First we need the notion of hyperbolic cubes. In the disc model of $H^{n}$ take coordinate hyperplanes $x_{i}=0$. Take a point $(t, \ldots, t)$, and all of its $2^{n}-1$ orthogonal projections to the intersections of coordinate hyperplanes. The convex hull of these $2^{n}$ points is a cube. The solid angle at the vertex $(0, \ldots, 0)$ is the spherical simplex with all dihedral angles $\frac{\pi}{2}$ (all-right simplex); the opposite solid angle is a fairly small regular spherical simplex, depending on $t$. The small angle is placed at the tip of the cubical cone, while the all-right angle is placed at the vertex corresponding to the barycenter of the $(n-1)$ simplex. 
We need to describe links of points in $X_{h}$. For this we need some notation. Let $C(x)$ denote the cube of $X$ containing $x$ in its interior. Denote by $\operatorname{Lk}(C(x) \subset X)$ the normal link of any point $c$ in the interior of $C(x)$ (which depends on $C(x)$ only) and by $L k(a \in A)$ the link of $a$ in $A$. Then we have

\section{Proposition 7.1.}

(1) Let $(x, h) \in X_{h}$. Then we have an isometry of links

$$
L k\left((x, h) \in X_{h}\right)=L k(C(x) \subset X) * L k\left(h \in C(x)_{h}\right) .
$$

Here $X * Y$ denotes the spherical join of piecewise spherical complexes $X, Y$ (see [BH p. 63]).

(2) If $X$ is locally $C A T(0)$ and $F_{S}$ is an extra retractible complex of groups, then the complex $X_{h}$ with its Moussong metric is locally $C A T(-1)$.

Proof. (1) is straightforward from the definition of the hyperbolization and the description of the metric.

(2) follows from (1) and the following three facts: the join of large links is large, the assumption that $X$ has large links and the fact that the Moussong metric has large links.

\section{Remarks.}

(1) The most notable defect of this hyperbolization is that it does not preserve the property of $X$ being a manifold for $\operatorname{dim} X \geq 3$. In dimension 3 this can be fixed by an explicit local resolution of singularities. In higher dimension such a resolution, preserving $C A T(-1)$ property is unknown to us.

(2) It is not really necessary to use cubical complexes which admit a folding map. If $F_{S}$ has a better functorial property, that is if a permutation of $S$ induces an automorphism $F_{S} \rightarrow F_{S}$, respecting the subgroup structure of $F_{S}$, then the complex $L_{S}$ is symmetric, and we can apply the hyperbolization to complexes without a folding map. The complex constructed in Section 6 has such a symmetry.

\section{Non right-angled groups}

Let $\left(m_{s t}\right)$ be a right angled Coxeter matrix with the set of indices $S$. Let $\left(m_{s t}^{f}\right)$ be any Coxeter matrix obtained from $\left(m_{s t}\right)$ by the following modification:

$$
\left(m_{s t}^{f}\right)= \begin{cases}m_{s t} & \text { if } m_{s t} \neq \infty \\ f_{s t} & \text { otherwise }\end{cases}
$$

with $f_{s t} \in\{5,6, \ldots, \infty\}$.

Proposition 8.1. Suppose the Coxeter group $(W, S)$ associated to $\left(m_{s t}\right)$ is Gromov hyperbolic. Then 
(1) The group $\left(W^{f}, S\right)$ associated to any of the matrices $m^{f}$ is Gromov hyperbolic.

(2) If the nerve $N(W, S)$ is connected, then $N\left(W^{f}, S\right)$ has the homotopy type of the wedge of $N(W, S)$ and a certain number of circles.

(3) If $\operatorname{vcd}(W) \geq 2$, then $\operatorname{vcd}(W)=\operatorname{vcd}\left(W^{f}\right)$.

Proof. To prove (1) we use Moussong's criterion for hyperbolicity: a Coxeter group is Gromov hyperbolic if none of its parabolic subgroups is affine of rank $\geq 2$ or splits into a product of two infinite parabolic subgroups.

If $T \subset S$, then hyperbolicity of $(W, S)$ implies that the Dynkin diagram of $W$ restricted to $T$ has at most one connected component with more than one point.

If the nontrivial connected component contains more than two points, the subgroup of $\left(W^{f}, S\right)$ spanned by $T$ is neither finite nor affine (as one sees by examining well known lists of finite and affine irreducible Coxeter groups) and does not split as a product of infinite groups.

On the other hand, if the nontrivial component has two points, or if the Dynkin diagram has no edges at all, the group spanned by $T$ in $W^{f}$ is either finite or contains an infinite dihedral subgroup of finite index. This proves (1).

To prove (2), consider the coarse simplicial structures on the nerves. Clearly $N(W, S)$ is a subcomplex of $N\left(W^{f}, S\right)$. Let $\tau$ be a simplex in $N\left(W^{f}, S\right)$ not in $N(W, S)$ and let $T \subset S$ denote the set of vertices of $\tau$. From the proof of Assertion (1) we know that the Dynkin diagram of $W^{f}$ restricted to $T$ has one connected component with two vertices say $t, s$ with $m_{s t}=\infty$ and $f_{s t}$ finite (while the other components of the Dynkin diagram are points). This implies that $\tau$ (in fact the edge $t s)$ is contained in the unique maximal simplex of $N\left(W^{f}, S\right)$, whose vertices are $t, s$ and all elements of $S$ commuting with both $t, s$. Thus either the edge $t s$ is itself a maximal simplex in $N\left(W^{f}, S\right)$ or its maximal simplex deformation retracts through its interior onto the union of its faces not containing $t s$, which is a subcomplex of $N(W, S)$. These deformation retractions can be performed independently and together they give a collapse of $N\left(W^{f}, S\right)$ onto the union of $N(W, S)$ and certain number of 1 -simplices. This proves $(2)$.

For the proof of (3) we need the following

Lemma 8.2. Let $K$ be a finite flag simplicial complex, having the property that for any simplex $\sigma$ in $K$ (including the empty simplex), $H^{i}\left(K_{\sigma} ; Z\right)=0$ for $i>k$, where $K_{\sigma}$ is the full subcomplex of $K$ spanned by the vertices not in $\sigma$. Then for any full subcomplex $L$ of $K$ we have $H^{i}(L ; Z)=0$ for $i>k$.

Proof. Let $V$ be the set of vertices of $K$, and let $L$ be spanned by $V-A \subset V$. We proceed by induction on cardinality of $A$. Note that for $|A|=0,1$ the conclusion follows from the assumptions.

Now let $|A| \geq 2$. If $A$ spans a simplex in $K$, we are done. Otherwise there are 
two vertices in $A$, say $v, w$, not connected by an edge in $K$. Consider subcomplexes $L_{v}, L_{w}, L_{v, w}$ of $K$ spanned by $V-(A-\{v\}), V-(A-\{w\}), V-(A-\{v, w\})$ respectively. Then $L_{v} \cap L_{w}=L$, and $L_{v} \cup L_{w}=L_{v, w}$. Now we have a piece of the Mayer-Vietoris sequence

$$
H^{i}\left(L_{v} ; Z\right) \oplus H^{i}\left(L_{w} ; Z\right) \rightarrow H^{i}(L ; Z) \rightarrow H^{i+1}\left(L_{v, w} ; Z\right)
$$

For $i>k$ groups on both left and right are zero by inductive assumption, hence the Lemma.

Now the proof of (3) in Proposition 8.1 proceeds as follows. By Assertion (2), the nerve $N\left(W_{S-T}^{f}, S-T\right)$ has the homotopy type of the wedge of $N\left(W_{S-T}, S-T\right)$ and certain number of circles. By the discussion in Section 1.4 and the Lemma, $H^{i}\left(N\left(W_{S-T}, S-T\right) ; Z\right)=0$ for $i>\operatorname{vcd}(W)$ and any $T$. Thus if $\operatorname{vcd}(W) \geq 2$, $H^{i}\left(N\left(W_{S-T}^{f}, S-T\right) ; Z\right)=0$ for $i>\operatorname{vcd}(W)$ and any $T$, and (3) follows.

\section{Complexity issue}

The complexes $L_{S}$ we construct have strikingly large complexity measured in terms of the number of either vertices or top dimensional simplices. Let $S_{n}, V_{n}$ denote respectively the number of simplices of dimension $n$ and of vertices in $L_{S}$, for $|S|=n+1$. Clearly $S_{n}=\left|F_{S}\right|$.

Since the link of any vertex $v$ in $L_{S}$ is isomorphic to $L_{S-s}, v$ is contained in $S_{n-1}$ simplices. Thus by double counting

$$
V_{n}=\frac{(n+1) S_{n}}{S_{n-1}}
$$

Since under the abelianization map $H_{s}^{S}$ surjects onto $Z_{2}^{S_{n-1}}$, we have

$$
S_{n} \geq S_{n-1} 2^{S_{n-1}} \quad \text { and } \quad V_{n} \geq(n+1) 2^{S_{n-1}} .
$$

Actual values of $S_{n}$ are probably much larger than predicted by these inequalities, making the growth of $S_{n}$ ridiculously fast. It may be desirable to improve this aspect of the construction.

On the other hand we would like to point out that flag no-square simplicial pseudomanifolds cannot be too simple, that is the number of their top simplices grows with the dimension significantly faster than exponentially.

Proposition 9.1. Let $v_{n}, s_{n}$ denote the minimal number of vertices and $n$-simplices respectively in a simplicial, flag, no-square pseudomanifold of dimension $n$. Then

$$
s_{n+1}>\frac{s_{n}^{2}}{n+2} \quad \text { and } \quad v_{n+1}>s_{n}
$$


Proof. Let $X$ be a simplicial, flag, no-square pseudomanifold of dimension $n+1$. Then the link of any simplex $\sigma$ in $X$ is also a simplicial, flag, no-square pseudomanifold of appropriate dimension. To prove this notice that since $X$ is flag, the join $\sigma * \operatorname{link}(\sigma)$ embeds in $X$ as the set of all top dimensional simplices containing $\sigma$. Thus a square in $\operatorname{link}(\sigma)$ is a square in $X$, and hence it has one of its diagonals in $X$. Since $X$ is flag, the diagonal is in the link.

By double counting, the number of $(n+1)$-simplices in $X$ is equal to $\frac{1}{n+2} \sum_{v}|l k(v)|$, where $v$ runs over all vertices of $X$, and $|l k(v)|$ denotes the number of $n$-simplices in the link of $v$.

Since links in $X$ are flag, no-square pseudomanifolds, we obtain the estimate

$$
s_{n+1} \geq \frac{v_{n+1} s_{n}}{n+2} .
$$

Now fix a vertex $x \in X$. Each $n$-simplex $\sigma$ in the link of $x$ determines the unique $(n+1)$-simplex in $X$ which contains $\sigma$ and is not contained in the star of $x$. Hence it determines a vertex of $X$ : the vertex of $\sigma$ not in the link of $x$. The vertices so obtained are all distinct by the no-square condition. Counting vertices in the star of $x$, and vertices obtained above we get

$$
v_{n+1} \geq 1+v_{n}+s_{n}
$$

Putting the two displayed inequalities together finishes the proof.

\section{References}

[B1] M. Bestvina, Virtual cohomological dimension of Coxeter groups, in: G. Niblo and M. Roller eds., Geometric Group Theory I, 19-23, LMS Lect. notes 181, Cambridge University Press, 1993.

[B2] M. Bestvina, http://www.math.utah.edu/ bestvina/.

[BH] M. Bridson and A. Haefliger, Metric spaces of Nonpositive Curvature, Grundlehren der Math. Wiss. 319, Springer, 1999.

[Bu] W. O. Bugaenko, Arithmetic crystallographic groups generated by reflections and reflective hyperbolic lattices, Lie groups, their discrete subgroups, and invariant theory, 33-55, Adv. Sov. Math. 8, AMS, Providence, 1992.

[D1] M. W. Davis, The cohomology of a Coxeter group with group ring coefficients, Duke Math. J. 91 (1998) no. 2, 297-314; Duke Math. J. 95 (1998) no. 3, 635.

[D2] M. W. Davis, Nonpositive curvature and reflection groups, in: R. Daverman and R. Shor eds., Handbook of Geometric Topology, Elsevier, 2001

[DJ1] M. W. Davis and T. Januszkiewicz, Hyperbolization of polyhedra, J. Diff. Geom. 34 (1991), 347-388.

[Dr] A. N. Dranishnikov, Boundaries of hyperbolic Coxeter groups, Topology and Applications 110 (2001), 29-38

[G] M. L. Gromov, Asymptotic invariants of infinite groups, in: G. Niblo and M. Roller eds., Geometric Group Theory II, LMS Lect. notes 182, CUP, 1993.

[GT] M. L. Gromov and W. P. Thurston, Pinching constants for hyperbolic manifolds, Inv. Math. 89 (1987), 1-12. 
[MS] L. Mosher and M. Sageev, Non manifold hyperbolic groups of high cohomological dimension. http://newark.rutgers . edu/ mosher

[M] G. Moussong, Hyperbolic Coxeter group, Ohio State University PhD thesis, 1987.

[S] R. Stanley, Combinatorics and commutative algebra, Birkhäuser, 1983.

[V] E. B. Vinberg, Hyperbolic reflection groups, Usp. Mat. Nauk 40 (1) (1985), 29-66.

T. Januszkiewicz and J. Świątkowski

Instytut Matematyczny UWr

pl. Grunwaldzki $2 / 4$

PL-50-384 Wroclaw

Poland

(Received: January 2, 2002)

(2) To access this journal online:

(20) http://www.birkhauser.ch 